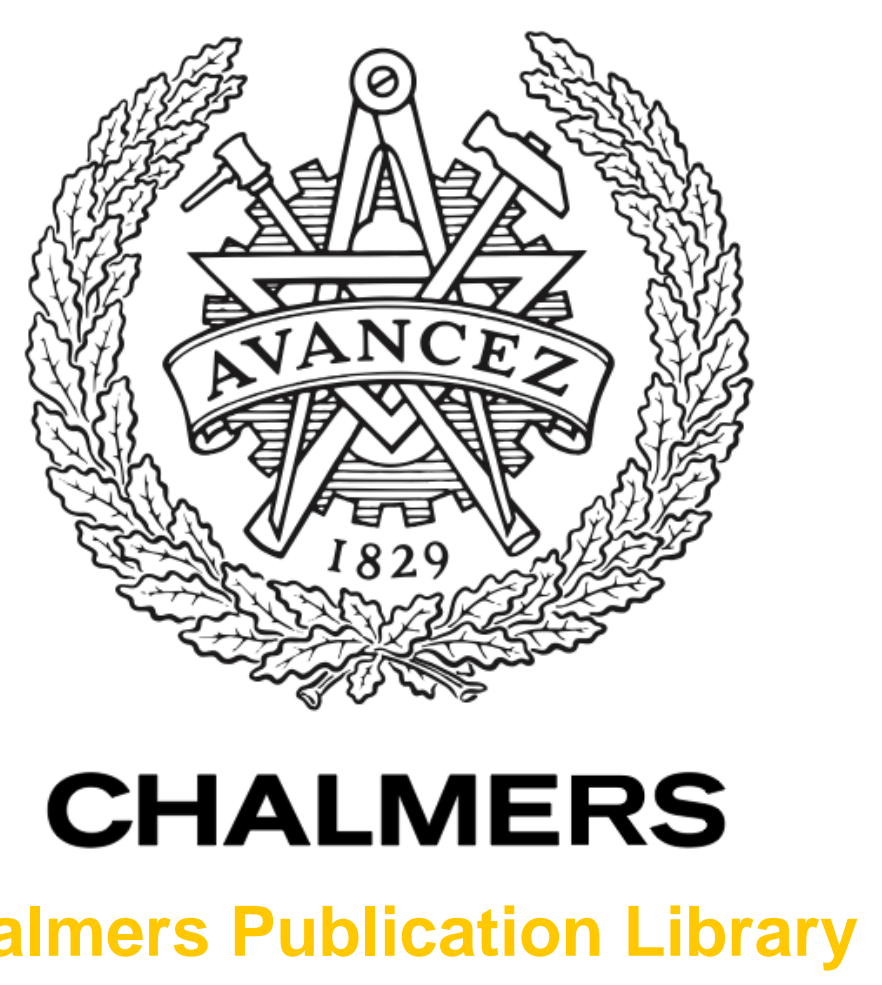

Chalmers Publication Library

\title{
Digital Predistortion for High Efficiency Power Amplifier Architectures Using a Dual-input Modeling Approach
}

This document has been downloaded from Chalmers Publication Library (CPL). It is the author's version of a work that was accepted for publication in:

IEEE Transactions on Microwave Theory and Techniques (ISSN: 0018-9480)

Citation for the published paper:

Cao, H. ; Mashad Nemati, H. ; Soltani Tehrani, A. (2012) "Digital Predistortion for High Efficiency Power Amplifier Architectures Using a Dual-input Modeling Approach". IEEE Transactions on Microwave Theory and Techniques, vol. 60(2), pp. 361-369.

http://dx.doi.org/10.1109/TMTT.2011.2176956

Downloaded from: http://publications.lib.chalmers.se/publication/148398

Notice: Changes introduced as a result of publishing processes such as copy-editing and formatting may not be reflected in this document. For a definitive version of this work, please refer to the published source. Please note that access to the published version might require a subscription.

Chalmers Publication Library (CPL) offers the possibility of retrieving research publications produced at Chalmers University of Technology. It covers all types of publications: articles, dissertations, licentiate theses, masters theses, conference papers, reports etc. Since 2006 it is the official tool for Chalmers official publication statistics. To ensure that Chalmers research results are disseminated as widely as possible, an Open Access Policy has been adopted.

The CPL service is administrated and maintained by Chalmers Library. 


\title{
Digital Predistortion for High Efficiency Power Amplifier Architectures Using a Dual-input Modeling Approach
}

\author{
Haiying Cao Student Member, IEEE, Hossein Mashad Nemati, Ali Soltani Tehrani Student Member, IEEE,,
} Thomas Eriksson, and Christian Fager Member, IEEE,

\begin{abstract}
In this paper, a novel model is proposed for dualinput high efficiency power amplifier (PA) architectures, such as envelope tracking (ET) and varactor-based dynamic load modulation (DLM). Compared to the traditional single-input modeling approach, the proposed model incorporates the baseband supply voltage/load control as an input. This advantage makes the new approach capable to achieve maximized average power-added efficiency (PAE) and minimized output distortion simultaneously. Furthermore, the new approach has shown to be robust towards time misalignment between the RF input and baseband supply voltage/load control signals, and it can be applied with a reducedbandwidth baseband supply voltage/load control.

Experiments have been performed in a varactor-based DLM PA architecture to evaluate the new modeling approach. The results show that it can achieve $9 \mathrm{~dB}$ and $7 \mathrm{~dB}$ better performance than the traditional approaches in terms of adjacent channel leakage ratio and normalized mean square error, respectively. At the same time, the average PAE is maximized. Similar results have been achieved with the proposed model even when reduced-bandwidth baseband load control signal is used or time misalignment between the RF and baseband load control input signals exists. Although the new approach is only tested with DLM architecture in this paper, it is very general and can be applied to ET architectures as well.
\end{abstract}

Index Terms-Dynamic load modulation, digital predistortion, envelope tracking, efficiency, linearization, power amplifier.

\section{INTRODUCTION}

$\mathbf{R}$ ECENTLY, various power amplifier (PA) architectures have been introduced to achieve high average poweradded efficiency (PAE) for modulated signals with high peakto-average power ratio (PAPR) [1]. Two of the most promising examples are envelope tracking (ET) [2], [3] and varactorbased dynamic load modulation (DLM) [4], as shown in Fig. 1. In these architectures, the DC supply and load impedance, respectively, are dynamically controlled along with the input power to maintain high efficiency over a wide range of output

This research has been carried out in the GigaHertz Centre in a joint research project financed by the Swedish Governmental Agency of Innovation Systems (VINNOVA), Chalmers University of Technology, Ericsson AB, Infineon Technologies AG, and NXP Semiconductors BV.

H. Cao and C. Fager are with the department of Microtechnology and Nanoscience, Chalmers University of Technology, Göteborg, Sweden email:[haiying,christian.fager]@ chalmers.se

H. M. Nemati is with Ericsson AB, Sweden email:[hossein.nemati]@ericsson.com

A. Soltani and T. Eriksson are with the department of Signals and Systems, Chalmers University of Technology, Göteborg, Sweden email:[asoltani,thomase]@chalmers.se

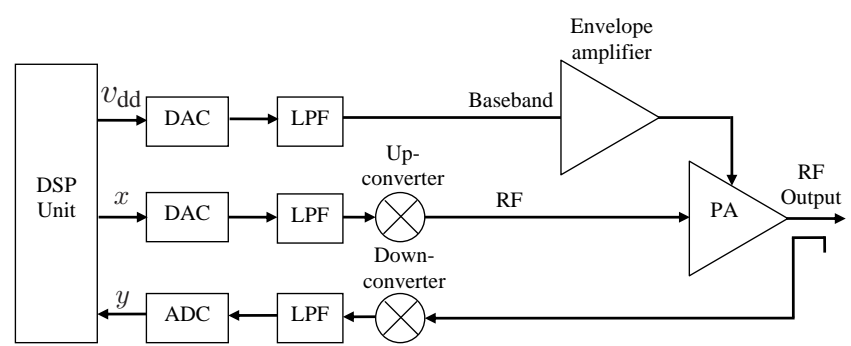

(a) Envelope tracking [3]

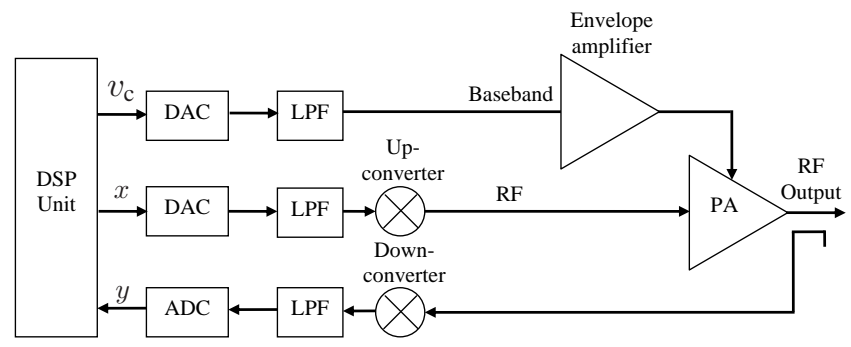

(b) Dynamic load modulation [10]

Fig. 1. Simplified block diagrams of high efficiency power amplifier architectures. $x$ is the RF input signal, $y$ is the RF output signal and $v_{\mathrm{dd}}$ and $v_{\mathrm{c}}$ are envelope signals for ET and DLM, respectively.

power levels. In this way, the average efficiency can be significantly improved compared to a traditional PA architecture. Details of how to co-control the RF and baseband envelope input signals to achieve optimal average PAE are discussed in [5], [6].

Despite that the ET and DLM PA architectures offer the capability to improve the average PAE, they have two important problems to be considered in practice. First, the bandwidth of the envelope signal is usually 3-4 times larger than that of the modulated RF signal [7]. This brings challenges for hardware designers to design a wideband DC/DC converter or tunable matching network with high efficiency. Second, the two input signals may be difficult to time-align properly [8]. Time-misaligned input signals can affect the linearity performance and degrade the efficiency [9]. Moreover, the dual-input nature of these architectures causes nonlinear distortion effects that need to be compensated for using special linearization techniques.

Dedicated linearization methods for either ET or DLM architectures have been proposed in [3], [10]-[14]. Results 
have shown that the nonlinearity introduced by the dynamic supply voltage or tunable matching network is possible to be compensated for by using digital predistortion techniques. A piecewise Volterra-based linearization method has been used for the ET architecture in [3], however, details on how to cocontrol the RF input and envelope signals to get maximized efficiency is not given. The linearization methods in [10]-[12] use static characterization results as a basis for an efficiencyoptimized co-control of the RF and envelope input signals. A conventional single-input digital predistorter (DPD) is then added to compensate for the residual memory effects and nonlinear distortion. However, the inaccuracies in the static models used in these methods will, as will be shown later in this paper, limit the overall linearization performance of transmitter. In [13], [14], nonlinear characteristics of a hybridenvelope elimination and restoration (EER) transmitter (similar to ET) have been fully investigated. A co-control scheme for the envelope and RF input signals and a new linearization configuration were developed. Experimental results have shown that improved efficiency performance has been achieved with good linearization results. For the bandwidth issue of envelope signal, a bandwidth reduction scheme is proposed in [7], and a 2-D look-up table approach is used to linearize the ET architecture with the reduced-bandwidth envelope signal. Despite the importance of these promising PA architectures and the research that has been done to linearize them, most of the linearization works are still in single-input-single-output sense. No dual/multi-input DPD linearization method has been presented to maximize efficiency and linearity performance, while considering the inevitable practical problems of envelope signal bandwidth reduction or time misalignment sensitivity in practical transmitters.

In this paper, a new linearization approach is proposed for high efficiency PA architectures with two signal paths, such as ET and DLM. Compared to the conventional singleinput linearization approaches used in the literature, the new dual-input model uses the envelope signal as an additional input to construct the predistorted RF input signal. We will theoretically show that the new approach can achieve better linearization and efficiency results, in particular when the bandwidth of the envelope signal and time misalignment problems are considered. Experiments are performed on a DLM PA architecture to evaluate the new linearization approach. Although the evaluation is done only on one of the mentioned PA architectures due to the availability of hardware, the new approach is general and can be applied to any other dual-input PA architecture similar to DLM, e.g., ET architecture. To the authors' knowledge, this paper presents, for the first time, a general and complete procedure for linearization of ET/DLM PA architectures

The paper is organized as follows. Section II presents the derivation of the proposed model. Section III presents the dualinput digital predistorter and its application with a reducedbandwidth envelope signal and time alignment. Experimental results are shown in Section IV and finally conclusions are drawn in Section V.

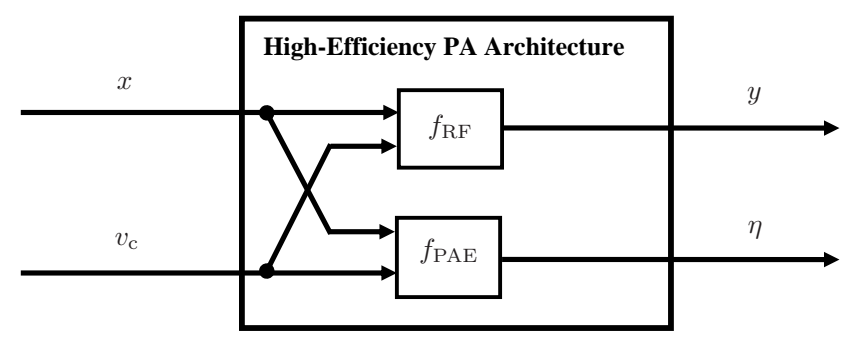

Fig. 2. A general model for high-efficiency power amplifier architectures. $x$ is the RF input signal, $v_{\mathrm{C}}$ is the envelope signal, $y$ is the output RF signal, $\eta$ is the instantaneous power-added efficiency, $f_{\mathrm{RF}}$ and $f_{\mathrm{PAE}}$ are transfer functions representing the RF path and output PAE path, respectively.

\section{Modeling of Dual-Input Power Amplifier ARCHITECTURES}

The dual-input characteristic of the ET and DLM PA architectures gives them one extra degree of freedom compared to traditional PA architectures. This allows them to enhance the average PAE and achieve better linearization performance at the same time, if proper design of the RF input and envelope signals is done. A general model representing the main concept of the ET and DLM PA architectures is shown in Fig. 2.

Mathematically, the output instantaneous PAE, $\eta$, and output RF signal, $y$, are functions of the RF input signal, $x$ (or its magnitude $|x|$ ), and the envelope signal, $v_{\mathrm{c}}$. The model equations can therefore be written as

$$
\begin{array}{r}
y=f_{\mathrm{RF}}\left(x, v_{\mathrm{c}}\right), \\
\eta=f_{\mathrm{PAE}}\left(|x|, v_{\mathrm{c}}\right) .
\end{array}
$$

Studying (1) and (2) carefully, we see that for a given RF output signal $y$, there can be many different combinations of $x$ and $v_{\mathrm{c}}$ that satisfy (1). Therefore, we have the freedom to choose the combination of $x$ and $v_{\mathrm{c}}$ that gives us the highest PAE among all possible solutions [6]. This leads to a constrained optimization problem of finding the optimal RF input signal $x_{\mathrm{opt}}$ and envelope signal $v_{\mathrm{c}, \mathrm{opt}}$, that can be expressed as

$$
\begin{array}{r}
\left(x_{\mathrm{opt}}, v_{\mathrm{c}, \mathrm{opt}}\right)=\underset{x, v_{\mathrm{c}}}{\operatorname{argmax}} f_{\mathrm{PAE}}\left(|x|, v_{\mathrm{c}}\right), \\
\text { subject to } f_{\mathrm{RF}}\left(x, v_{\mathrm{c}}\right)=y_{\text {desired }}
\end{array}
$$

where $y_{\text {desired }}$ is the desired output signal of the PA. It can be seen that the maximum PAE and minimum distortion can be achieved by jointly finding the optimal RF input and optimal envelope signals. This is the basis of the static model used for the existing linearization methods [10]-[12], which will be discussed further in the following subsection.

\section{A. Review of Previous Works}

Many dedicated linearization papers have been published in recent years for high-efficiency PA architectures, of which all rely on a static model [10]-[12]. Interestingly, for the outphasing PA in [15] which has two RF inputs, a static model similar to the one in [10] is used as parts of the linearization method. All the dedicated linearization methods may be represented by either Fig. 3 or Fig. 4, where $f_{1}$ and $f_{2}$ 


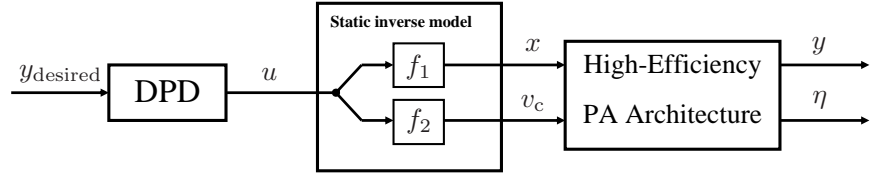

Fig. 3. Linearization method with a static model for high-efficiency PA architectures [10], [12].

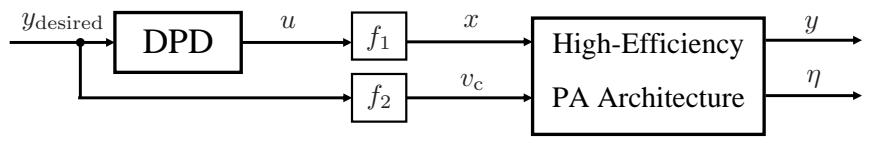

Fig. 4. Modified linearization method for high-efficiency PA architectures [11], [12]

are static polynomial functions for the RF input and envelope signals, respectively.

The static model in [10]-[12] is basically derived by the following steps.

- Perform static power sweep measurements

- Identify the efficiency-optimized settings from (3) based on the measurements

- Polynomial fitting models are used to construct the RF input and envelope signals with the efficiency-optimized settings

As can be noticed, the static model has some disadvantages. In practice, the static model can only invert the nonlinearity of the PA architectures to a limited extent and increasing the order of the polynomial model may lead to over-fitting problems, due to limited number of measurement points. Most importantly, there exist fitting errors at both the RF and baseband branches. The DPD presented in Fig. 3 cannot compensate for these fitting errors, while the DPD in Fig. 4 can only compensate for the distortion at the RF branch, as it has no knowledge of the errors at the baseband branch. Errors at the baseband branch will, however, lead to reduced linearity of the RF output signal. The normal single-input model approach of the existing linearization methods as shown in Fig. 3 and 4 cannot therefore obtain optimal efficiency and linearity results simultaneously.

\section{B. Derivation of A New Model}

In this paper, a new model is proposed for ET and DLM PA architectures. The new model does not depend on the static model where the RF and baseband branches are separated, as shown in Fig. 3 and 4. On the other hand, for the new model, the predistorted signal at the RF branch is constructed using the information at both the baseband and RF branches. Moreover, the new model adopts a two-step approach, instead of solving (3) for the optimal RF and envelope input signals jointly. The first step is to choose the envelope signal that can maximize the average PAE. The second step is to choose the $\mathrm{RF}$ input signal which minimizes the distortion between the RF output signal $y$ and the desired RF output signal $y_{\text {desired. }}$. As we will show, this two-step approach does not imply any loss of generality and it is still an optimal design. The basic idea of our discussion in the following is that for any given envelope signal $v_{\mathrm{c}}$, the PA can be properly predistorted as long as the DPD has knowledge of the chosen $v_{\mathrm{c}}{ }^{1}$ It is true that for some choices of $v_{\mathrm{c}}$ the linearization may be more difficult, but we argue that the linearization can still be done with high accuracy for a large class of $v_{\mathrm{c}}$, and this statement is supported by extensive measurement results later in Section IV.

If an appropriate envelope signal $v_{\mathrm{c}}$ is given, the function $f_{\mathrm{RF}}$ in (1) can be seen as a normal transfer function for RF PAs with a one-to-one mapping between the RF input signal $x$ and output signal $y$. Hence, according to the $p$-th order inverse theory by Schetzen [16], the function $f_{\mathrm{RF}}$ is normally invertible, and the predistorted RF input signal can be written as

$$
x=f_{\mathrm{RF}}^{-1}\left(y, v_{\mathrm{c}}\right)
$$

where $f_{\mathrm{RF}}^{-1}$ is the inverse function with respect only to $x$ and $y$, for a known $v_{\mathrm{c}}$. Note that the possibility to predistort a PA as in (5) is generally true even for a non-optimally chosen $v_{\mathrm{c}}$, e.g., $v_{\mathrm{c}}$ may be constant or bandlimited. Using (5) with $y=$ $y_{\text {desired, }}$, we can derive the RF signal fulfilling the condition in (4) which can be expressed as

$$
x_{\mathrm{opt}}=f_{\mathrm{RF}}^{-1}\left(y_{\text {desired }}, v_{\mathrm{c}}\right) \text {. }
$$

Until now, we have derived the optimal RF input signal $x_{\mathrm{opt}}$ which is optimized for linearization performance. However, the efficiency is not optimized yet, i.e. the envelope signal, $v_{\mathrm{c}}$, is a non-optimally chosen signal.

In order to maximize the efficiency, by replacing the variable $x$ in (2) with $x_{\text {opt }}$ using (6), the efficiency-optimized envelope signal $v_{\mathrm{c}, \mathrm{opt}}$ can be derived as

$$
\begin{aligned}
v_{\mathrm{c}, \mathrm{opt}} & =\underset{v_{\mathrm{c}}}{\operatorname{argmax}} f_{\mathrm{PAE}}\left(\left|x_{\mathrm{opt}}\right|, v_{\mathrm{c}}\right) \\
& =\underset{v_{\mathrm{c}}}{\arg { }_{\mathrm{PAE}}}\left(\left|f_{\mathrm{RF}}^{-1}\left(y_{\text {desired }}, v_{\mathrm{c}}\right)\right|, v_{\mathrm{c}}\right) .
\end{aligned}
$$

From (7), we can see that the optimal envelope signal $v_{\mathrm{c}, \mathrm{opt}}$ giving maximum efficiency of the PA architecture is only a function of the desired output signal $y_{\text {desired }}$, and can thus be formulated as

$$
v_{\mathrm{c}, \mathrm{opt}}=g\left(y_{\text {desired }}\right) .
$$

We should notice that although the runtime expression for $v_{\mathrm{c}, \text { opt }}$ can be formulated in such a simple way, the identification of the optimal function $g$ can be quite difficult, as is obvious from (7). In the next section, more details about the identification of the function $g$ will be presented.

Once the optimal envelope signal $v_{\text {c,opt }}$ is obtained, the optimal predistorted RF input signal can be resolved directly from (6). With these two optimal-design signals, the optimal average PAE and high linearity can be achieved at the same time. A block diagram representing this new model is shown in Fig. 5.

The main advantage with this proposed model is that it solves the optimization of efficiency and the minimization of distortion in separate steps, while still being fully general.

\footnotetext{
${ }^{1}$ There are, of course, some choices of $v_{\mathrm{c}}$ which make linearization impossible, but those choices can be avoided in the design, as outlined later For a large class of envelope signals $v_{\mathrm{c}}$, accurate linearization is indeed possible.
} 


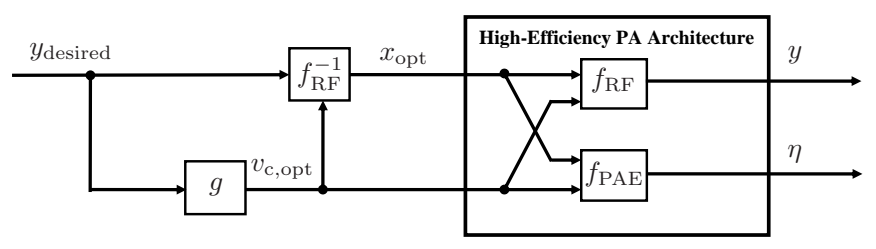

Fig. 5. The new model for linearizing high-efficiency PA architectures.

This makes the derivation of the optimal functions $g$ and $f_{\mathrm{RF}}^{-1}$ much simpler, and the linearization results are far better than those of previously proposed methods as will be shown in the experimental results.

\section{The New Dual-InPut Linearization Method}

Based on the derivation in Section II, a new dual-input linearization method is presented in this section. In practice, the ET and DLM PA architectures have the envelope signal bandwidth and time misalignment problems that need to be addressed as mentioned previously. Therefore, the design of the dual-input linearization method should be able to take into account these considerations as well.

\section{A. Behavioral Model of Efficiency-Optimized Function}

The efficiency-optimized function $g$ in Fig. 5 (or in (8)) can be modeled by a polynomial function as

$$
v_{\mathrm{c}}(n)=g(y(n))=\sum_{k=0}^{K} a_{k}|y(n)|^{k},
$$

where $y$ is the RF output signal, $a_{k}$ is the model coefficient and $K$ is the nonlinear order. To identify the optimal function $g$ or extract the model coefficients a, static CW measurements are first utilized to sweep both the amplitudes of the RF and envelope input signals. Static output amplitudes and efficiencies are then recorded. At each output power level, the combination of input power and envelope input signal that results in highest efficiency is used. This procedure guarantees that the PA can be linearized, since the efficiency-optimized envelope signal, $v_{\mathrm{c}, \mathrm{opt}}$, is chosen among the set of $v_{\mathrm{c}}$ that can generate the desired output power. The resulting efficiency maximized relationship between the envelope voltage and the output power is implemented and used as the optimal function $g$. It should be noted that, since in our case static $\mathrm{CW}$ measurements rather than modulated measurements are used to identify the optimal function $g$, it is not guaranteed that maximum efficiency is obtained. However, in our experience, and for the power levels considered in this work, the degradation of efficiency is negligible. The linearity is not compromised by the choice of $g$ either, since the envelope signal $v_{\mathrm{c}}$ is used in generation of the RF input signal in (6).

\section{B. Behavioral Model of Linearity-Optimized Function}

The function $f_{\mathrm{RF}}^{-1}$ in Fig. 5 can be seen as a dual-input single-output behavioral model for the high-efficiency PA architectures. The dual-input model can be extended from regular single-input single-output RF PA behavioral models by adding one real-valued dimension corresponding to the envelope signal. As we know, the generalized memory polynomial (GMP) model [21] is known to have excellent performance in terms of linearity versus complexity [22]. In this work, considering the complexity issue, a modified 2-D GMP model is developed based on the original GMP model and used for the dual-input single-output behavioral model. The digital predistortion function $f_{\mathrm{RF}}^{-1}$ can then be written as

$$
\begin{aligned}
& x(n)=f_{\mathrm{RF}}^{-1}\left(y(n), v_{\mathrm{c}}(n)\right)= \\
& \sum_{n_{1}=1}^{N_{1}} \sum_{m_{1}=0}^{M_{1}} \sum_{m_{v}=0}^{M_{v}} \sum_{p=0}^{P} h_{n_{1} m_{1} m_{v}, p} y_{m_{1}}\left|y_{m_{1}}\right|^{n_{1}} v_{\mathrm{c}, \mathrm{m}_{\mathrm{v}}}^{p} \\
& +\sum_{n_{2}=1}^{N_{2}} \sum_{m_{2}=0}^{M_{2}} \sum_{l_{1}=1}^{L_{1}} \sum_{m_{v}=0}^{M_{v}} \sum_{p=0}^{P} h_{n_{2} m_{2} l_{1} m_{v}, p} y_{m_{2}}\left|y_{m_{2}+l_{1}}\right|^{n_{2}} v_{\mathrm{c}, \mathrm{m}_{\mathrm{v}}}^{p} \\
& +\sum_{n_{3}=1}^{N_{3}} \sum_{m_{3}=0}^{M_{3}} \sum_{l_{2}=1}^{L_{2}} \sum_{m_{v}=0}^{M_{v}} \sum_{p=0}^{P} h_{n_{3} m_{3} l_{2} m_{v}, p} y_{m_{3}}\left|y_{m_{3}-l_{2}}\right|^{n_{3}} v_{\mathrm{c}, \mathrm{m}_{\mathrm{v}}}^{p}
\end{aligned}
$$

where $h$ are the model coefficients for the 2-D GMP model, $N$ is the nonlinear order, $L$ is the lagging/leading memory depth, $M$ is the RF input signal memory depth, $P$ is the nonlinear order for the envelope signal $v_{\mathrm{c}}, M_{v}$ is the envelope signal memory depth, $y_{m}$ is the RF output signal with delay $m, y(n-$ $m)$, and $v_{\mathrm{c}, \mathrm{m}_{\mathrm{v}}}$ is the envelope signal with delay $m_{\mathrm{v}}, v_{\mathrm{c}}(n-$ $\left.m_{v}\right)$.

This new behavioral model has one more input-the envelope signal-than the normal single-input PA behavioral models. In this case, the knowledge of the envelope signal can be used by the dual-input model when constructing the predistorted RF input signal. Thus, even if there exist fitting errors when deriving the optimal envelope signal, this dual-input model can take into account those effects and compensate for them through the predistorted RF signal. This advantage enables the proposed model to have the potential to outperform existing linearization methods for ET and DLM PA architectures. In regards to the identification of this behavioral model, although having an extra input compared to traditional single-input models, it can be noticed that the output is still linear with respect to the model coefficients. Therefore, the conventional least squares method can still be used to identify the dual-input behavioral model.

In practical implementations, the RF and baseband branches have different delays [9], [17]. If the predistorted RF input and envelope signals are not time aligned, even in the scale of less than one integer sample, the linearity and efficiency will be seriously affected [17]. Hence, fractional sample delay estimation is required in order to achieve the best performance offered [9]. The new dual-input behavioral model in this paper can, however, relax the time delay estimation requirement, since the envelope signal with memory can be included in the model as an input. In this case, integer sample delay estimation is enough. This can be verified by the experimental results in the next section. 


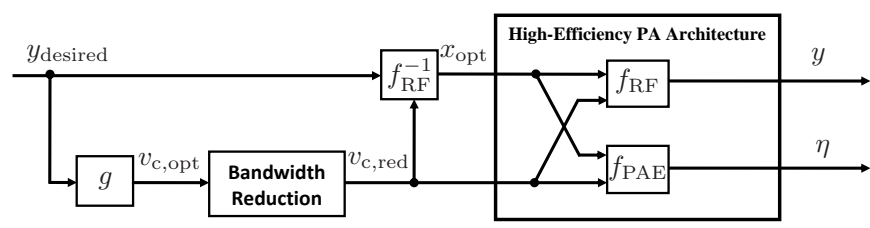

Fig. 6. The new linearization method for high-efficiency PA architectures with bandwidth reduction for envelope signal $v_{\mathrm{c} \text {,opt }}$.

\section{Bandwidth Reduction of The Envelope Signal}

The bandwidth expansion of the envelope signal in ET/DLM PA architectures is creating severe practical challenges for the hardware design of the DC/DC converter in ET or the tunable matching network in DLM. In particular for ET, there is a direct trade-off between the envelope signal bandwidth and envelope amplifier efficiency [7]. Different methods have, therefore, been proposed to reduce the bandwidth of the envelope signal for ET architecture [7], [18], [19]. The results shown in [7] is promising, they reported that the bandwidth of the envelope signal can be reduced from $20 \mathrm{MHz}$ to 5 $\mathrm{MHz}$, while the average PAE is reduced from $54.1 \%$ to $49.5 \%$. The existing linearization methods in [10], [11] cannot be directly used with reduced-bandwidth envelope signal, since the predistorted RF input signal does not have the knowledge of the envelope signal. Hence, when the characteristics of the envelope signal change (e.g., the envelope signal is bandwidthreduced), the existing linearization methods are not optimal for linearity any more.

Recently, authors in [20] have proposed a linearization method which employs the reduced-bandwidth envelope signal as an input. Their measurement results have shown that the pronounced memory distortion is possible to compensated for by such a linearization method. However, since no memory terms are used for either the RF or the envelope signals in their linearization method, some memory effects are still present and the linearization performance is limited.

In this paper, we incorporate the bandwidth reduction scheme in [7] with the new linearization method. The block diagram can be easily modified by adding a bandwidth reduction block to Fig. 5, as shown in Fig. 6. The resulting reduced-bandwidth envelope control signal and its spectrum are shown in Fig. 7, where the reduced-bandwidth of the envelope signal is chosen to be $5.6 \mathrm{MHz}^{2}$ as an example. The beauty of the proposed method is that the bandwidth reduction can be directly incorporated as part of the normal operation of the linearization, without any structural changes.

As we know, the reduced-bandwidth envelope signal may introduce additional memory effects to the PA [7], [20]. However, the dual-input DPD can be used to mitigated these memory effects as the predistorted RF input signal is now constructed by the reduced-bandwidth envelope signal with memory in (10). In other words, the constructed predistorted RF input signal is still optimal in terms of linearity. The average PAE, on the other hand, may be degraded since the reduced-bandwidth envelope signal is not the efficiency-

\footnotetext{
${ }^{2}$ The bandwidth is defined by $99.99 \%$ of the signal energy.
}

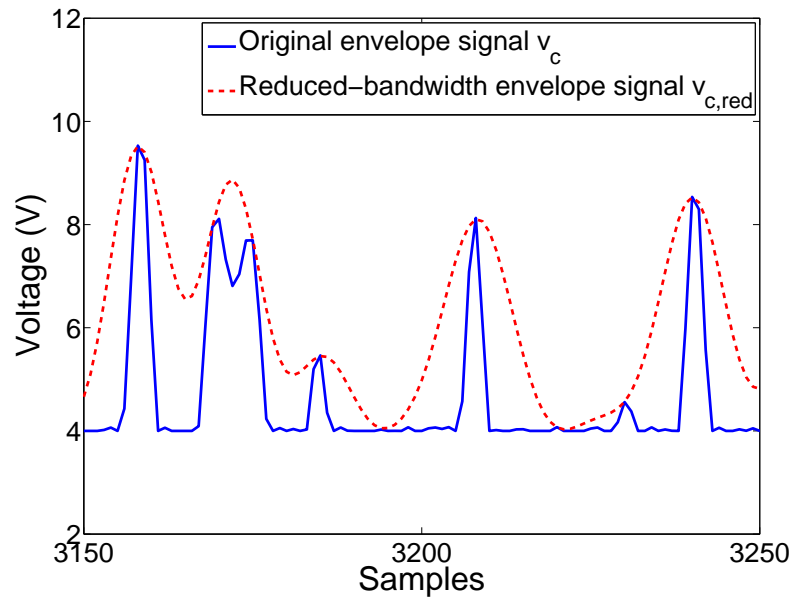

(a)

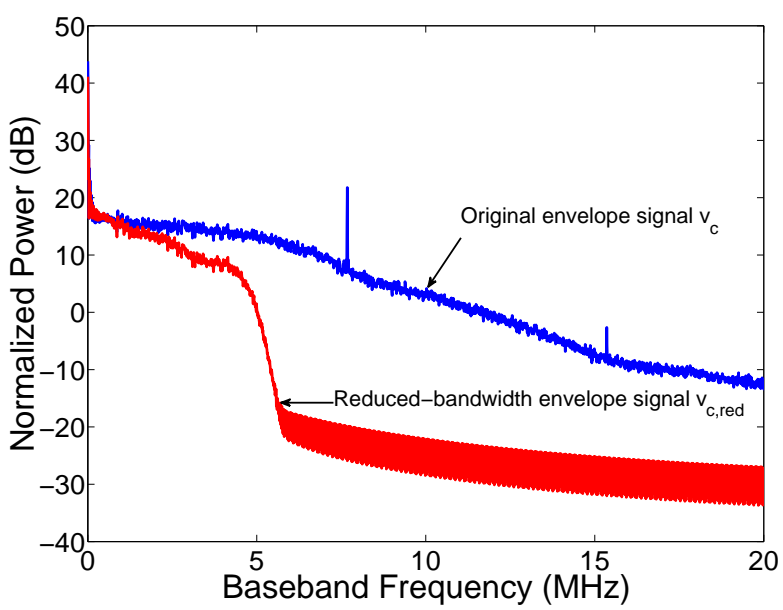

(b)

Fig. 7. Time and frequency domain plots of the original and reducedbandwidth envelope signals for a $3.84 \mathrm{MHz}$ single carrier WCDMA signal. The bandwidths of the original and reduced-bandwidth envelope signals are 12.5 MHz and 5.6 MHz, respectively. (a) Time domain waveforms of the reduced-bandwidth envelope signal $v_{\mathrm{c}, \text { red }}$ and original envelope signal $v_{\mathrm{c}}$. (b) Power spectra of $v_{\mathrm{c}, \text { red }}$ and $v_{\mathrm{c}}$.

optimized signal any more, but rather a slow time-varying function of it.

\section{Complexity}

In terms of complexity, the number of coefficients of the proposed dual-input model increases with the nonlinear order $P$ and memory term $M_{v}$ of the envelope signal. Therefore, it has $M_{v} \times(P+1)$ more coefficients than a regular DPD, as the one used in [10], [11]. However, experimental evaluation has shown that only low orders of $P$ and $M_{\mathrm{v}}$ are needed $\left(0 \leq P \leq 2\right.$ and $\left.0 \leq M_{\mathrm{v}} \leq 2\right)$ to get good linearization results with the new model. The added complexity of the new model is, therefore, acceptable considering its large performance improvements.

\section{EXPERIMENTAL RESUltS}

In this section, measurements are performed to evaluate the new linearization method. The measurement setup is 


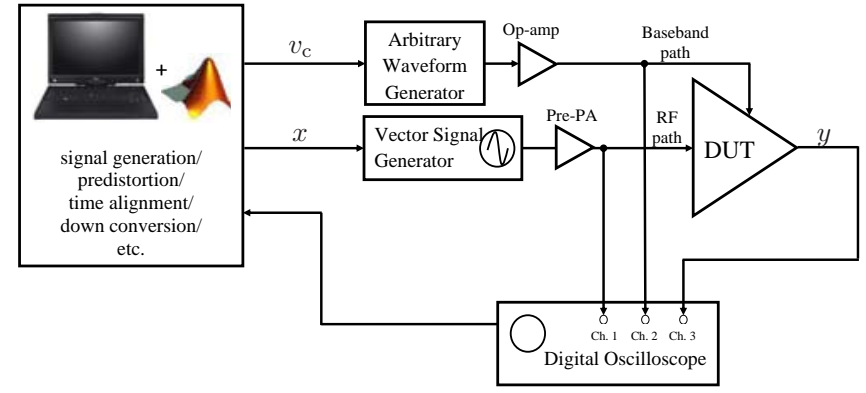

Fig. 8. Measurement setup with a dynamic load modulation power amplifier architecture as the DUT.

shown in Fig. 8, where an Agilent E4438C vector signal generator is used to generate the RF input signal, a Tabor electronics WW2572A arbitrary waveform generator (AWG) is used to generate the envelope signal and an Agilent 54845A oscilloscope is used as a receiver to capture the RF output signal. The device under test (DUT) is a varactor-based DLM PA architecture, operating at $2.65 \mathrm{GHz}$ [11]. The peak output power is around $38 \mathrm{dBm}$. In order to achieve the full dynamic swing of the envelope voltage, a LM 7171A op-amp from National Semiconductor Corporation is used also. The signal used is a single carrier WCDMA signal with $7 \mathrm{~dB}$ PAPR. Time alignment between the RF and envelope input signals has been performed using the technique in [17] before they are uploaded.

Four different experimental scenarios have been defined to evaluate the feasibility of the proposed dual-input linearization method. First, normal operation of the DUT is used, i.e. no time misalignment and full envelope signal bandwidth are used. In the second scenario, the linearization and efficiency performance with different reduced-bandwidths of the envelope signal are investigated. In the third scenario, different time misalignments between the RF and envelope input signals are tested. In the final scenario, time misaligment sensitivity of the new model is investigated with full and reduced envelope signal bandwidths.

\section{A. Normal Operation}

In the experiment, we have tested with different model orders. Finally, we select the nonlinear order 7 and memory depth 4 for the RF input signal which gives the best performance. In the following experiments, these orders for the RF input signal are used if not otherwise specified. Note that, the memory depth of the envelope signal is set to zero for this application and only nonlinear order $P=1$ is used, since the $\mathrm{RF}$ input and envelope signals are already time aligned and full bandwidth envelope signal is used.

Fig. 9 shows the AM/AM and AM/PM of the proposed dual-input DPD. The performance of the other linearization techniques proposed in literature are included as references for comparison. We can clearly see that the proposed DPD has better performance than all other tested linearization methods. The residual gain and phase error, after linearization with the new model, is less than $0.5 \mathrm{~dB}$ and 2 degrees, respectively. This indicates that the envelope signal as an additional input to

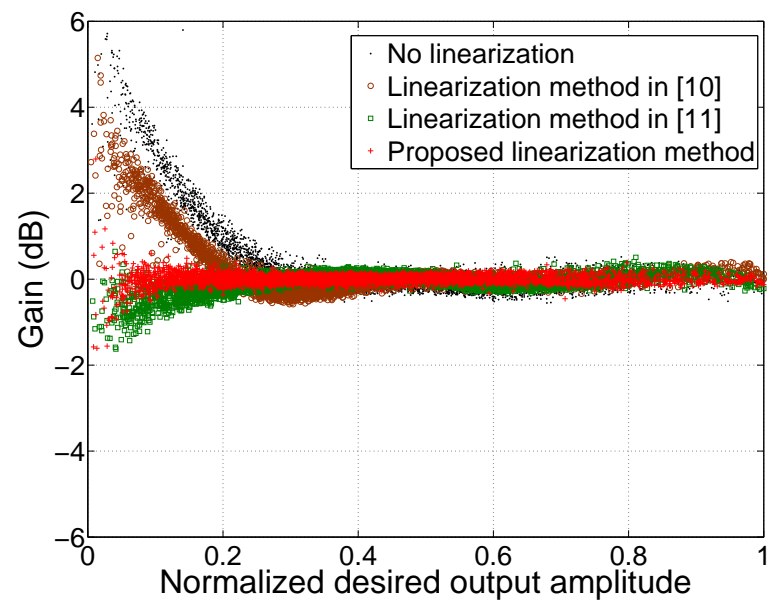

(a) $\mathrm{AM} / \mathrm{AM}$

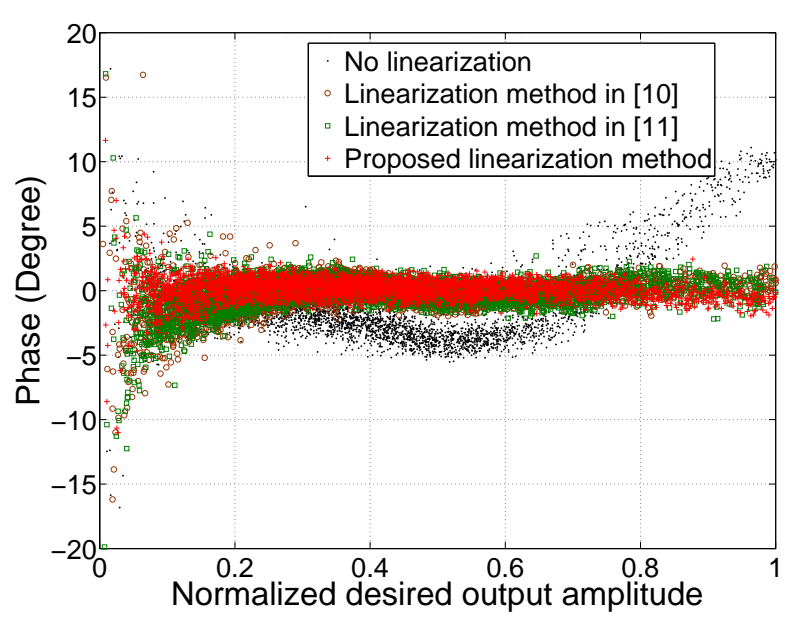

(b) $\mathrm{AM} / \mathrm{PM}$

Fig. 9. $\mathrm{AM} / \mathrm{AM}$ and $\mathrm{AM} / \mathrm{PM}$ performance when different linearization methods are applied.

the DPD can indeed help to improve the linearity performance. The reason is that the polynomial fitting errors in $v_{\mathrm{c}}$ are taken into consideration by the dual-input model and compensated for when generating an optimal RF input signal that linearizes the PA. Fig. 10 shows the linearized output spectra of the proposed method and the techniques in [10], [11]. It is noticed that the spectrum of the proposed method is already very close to the noise floor of the measurement setup which is the linearity performance limits.

Table. I summarizes the comparison of the different linearization methods. Note that the calculation of PAE does not include the power consumption of the LM 7171A opamp, since it is negligible in the experiments. The results demonstrate that the proposed linearization method can be used effectively to minimize the distortion and maximize the average PAE simultaneously for high-efficiency PA architectures. This is due to that the envelope signal is optimized for efficiency, while the predistorted RF input signal is optimized for linearity at the same time. Concerning the complexity of the proposed linearization method, it has twice the number 
TABLE I

Comparison of Modulated Measurement Results with Different Linearization Methods

\begin{tabular}{|c|c|c|c|c|c|}
\hline Methods & $\begin{array}{c}\text { Average PAE } \\
{[\%]}\end{array}$ & $\begin{array}{c}\text { Average } \\
\text { Output Power } \\
{[\mathrm{W}]}\end{array}$ & NMSE [dB] & ACLR1 [dB] & $\begin{array}{c}\text { Number of } \\
\text { Coefficients }\end{array}$ \\
\hline Linearization method in [10] & 48 & 1.2 & -32 & -41 & 100 \\
\hline Linearization method in [11] & 49 & 1.2 & -34 & -44 & 100 \\
\hline Proposed linearization method & 49 & 1.2 & -39 & -50 & 200 \\
\hline
\end{tabular}

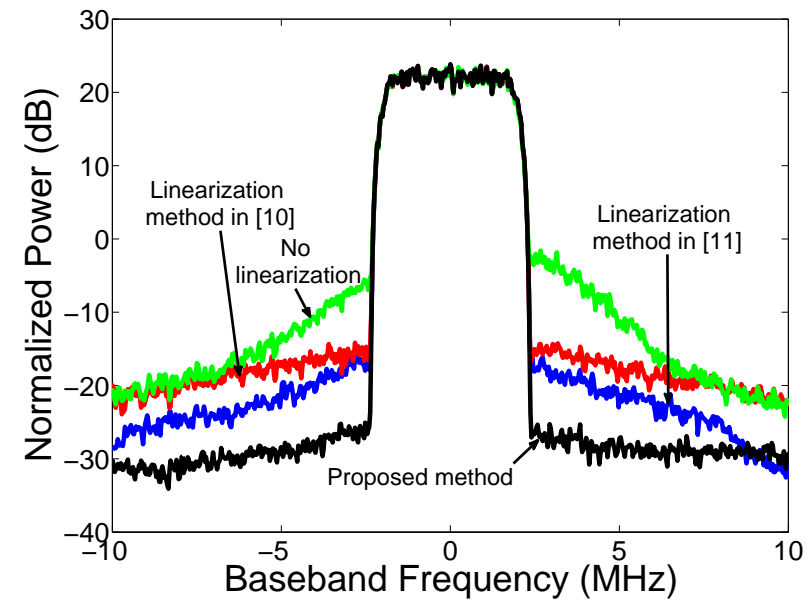

Fig. 10. Output power spectra when different linearization methods are applied.

of coefficients compared to the existing linearization methods. However, considering the improvement of performance and if a much less complex behavioral model for the DPD is used, the proposed linearization method is a promising candidate for the high-efficiency PA architectures having two inputs.

\section{B. Reduced-Bandwidth Envelope Signal}

In the second experiment, we evaluate the effectiveness of the new dual-input DPD when the envelope signal bandwidth is reduced. This experiment also demonstrates the generality of the proposed method. The memory terms of the envelope signal are used in this application to compensate for the additional memory effects introduced by the reduced-bandwidth envelope signal and is set to $M_{v}=2$. Fig. 11 shows the ACLR and average PAE vs. different envelope signal bandwidths. The results indicate that the proposed DPD can achieve good linearization results even when the bandwidth of the envelope signal is reduced from $12.5 \mathrm{MHz}$ to $2 \mathrm{MHz}$. At the same time, the average PAE is only degraded by less than $3 \%$. It should be noted that the existing linearization methods in [10], [11] cannot be successfully used for the reduced-bandwidth envelope signal. As shown in Fig. 11, when the envelope signal bandwidth is reduced, the ACLR of the linearization method in [11] becomes worse. When the envelope signal bandwidth is less than $5.6 \mathrm{MHz}$, the linearity degrades more severely. Also, the average PAE of the linearization method in [11] is worse than that of the proposed model. These results prove that the additional envelope signal input to the DPD can indeed

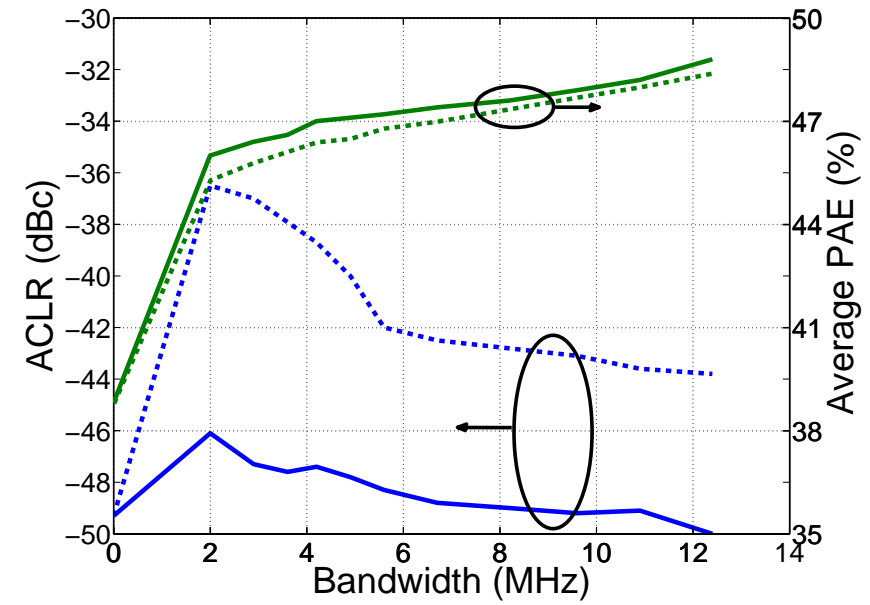

Fig. 11. Average PAE and ACLR vs. bandwidth of the envelope signal $v_{\mathrm{c}}$. Solid lines denote the proposed linearization method; dashed lines denote the linearization method in [11].

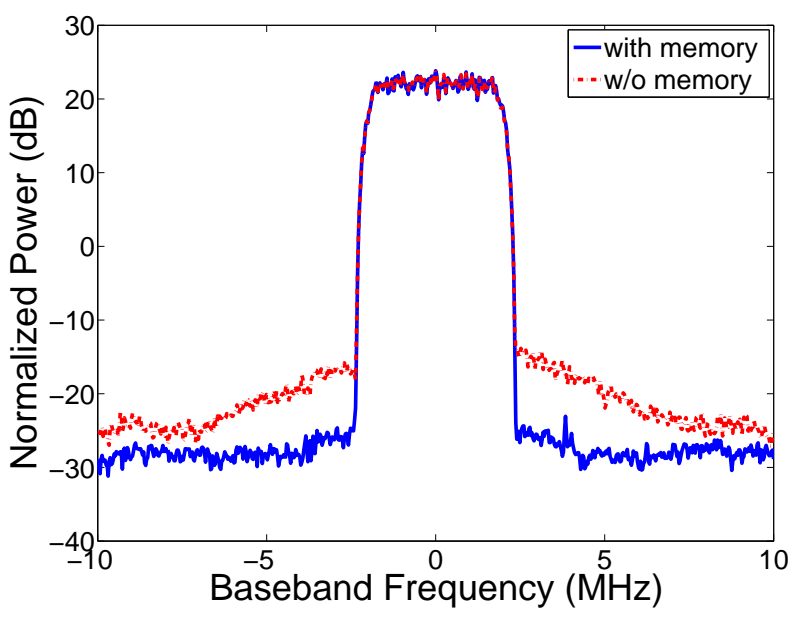

Fig. 12. Output power spectra of the proposed method with and without memory compensation. With memory compensation, the memory depths $M$ and $M_{v}$ used for the RF input signal and envelope signal are 4 and 2, respectively.

improve the linearization performance, even the property of the envelope signal changes.

The zero bandwidth of the envelope signal is also included in Fig. 11, which corresponds to traditional PA operation with DLM disabled. In this case, we can see that the average PAE drops severely, while the linearization results become better. This is the expected behavior, since this case corresponds to the normal single-input single-output PA and DPD operations. 


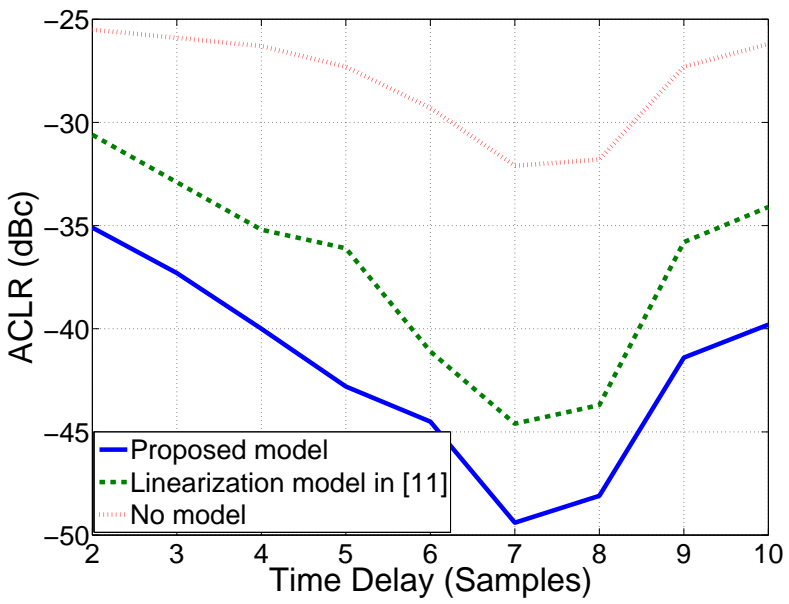

Fig. 13. ACLR vs. delay samples between the predistorted RF input signal $x$ and envelope signal $v_{\mathrm{C}}$.

As stated previously in Section III, a reduction of the envelope signal bandwidth will result in severe memory effects. In order to justify the need for memory compensation in the DPD, the proposed DPD has therefore also been evaluated without any memory terms in the RF and envelope signals, i.e. $M=M_{v}=0$ in (10). In this experiment, the bandwidth of the reduced-bandwidth envelope signal is $6.7 \mathrm{MHz}$. The output power spectra with and without memory compensation are shown in Fig. 12. From the output spectra, it can be clearly noticed that the ACLR is only around $-40 \mathrm{dBc}$ when there is no memory compensation in the DPD, the nonlinear distortion can be further suppressed by around $9 \mathrm{~dB}$ by including the memory terms. This indicates that the memory compensation in the DPD is necessary for the linearization of practical high-efficiency PA architectures with finite envelope signal bandwidths.

\section{Time Misalignment}

Different time delays between the RF and baseband branches are manually added in the DUT in order to test the sensitivity to time misalignment when the new linearization method is used. As mentioned before, the memory depth $M_{v}$ of the envelope signal is set to 2 throughout these experiments. Fig. 13 shows the performance of the proposed DPD with different time delays. We can see that for an ACLR requirement of $-45 \mathrm{dBc}$, which is the standard for the single carrier WCDMA signal [23], the time delay estimation requirement can be significantly relaxed compared to the existing linearization methods. In fact, a fractional time delay estimation is not needed and at least one sample delay mismatch is still tolerable. This can further reduce the complexity of the transmitter implemented, since the sincinterpolation for estimating the fractional time delay [17] can thus be avoided.

\section{Reduced-Bandwidth and Time Misalignment}

In the final experiment, the proposed dual-input DPD is used to linearize a DLM PA when both time mismatches and

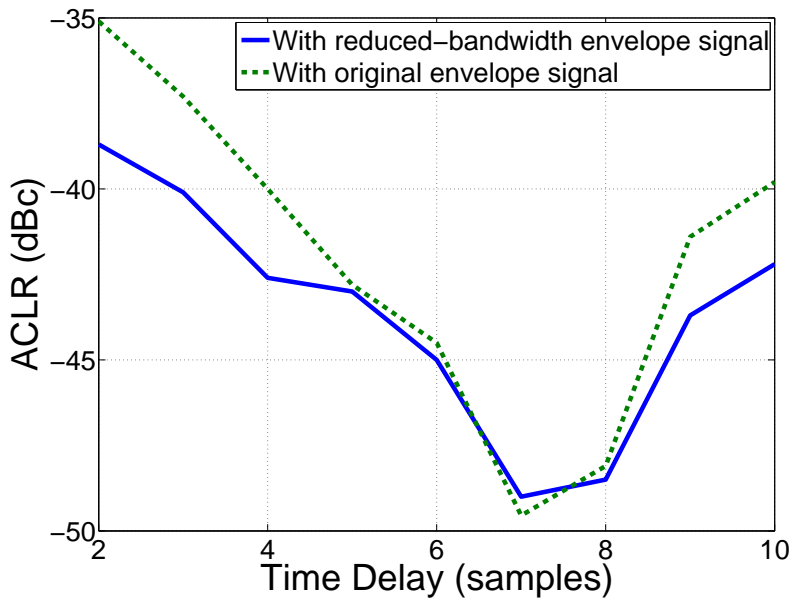

Fig. 14. ACLR vs. delay samples between the predistorted RF input signal $x$ and reduced-bandwidth envelope signal $v_{\mathrm{c}, \text { red }}$.

reduced-bandwidth envelope signal is used. The bandwidth of the envelope signal in this experiment is reduced to around 6.7 MHz. Fig. 14 shows that if there is time mismatches, the linearization performance with the reduced-bandwidth envelope signal is better than the case in which full envelope signal bandwidth is used. The reason is that when the bandwidth of the envelope signal is reduced, the envelope changes more slowly. In this case, the model becomes less sensitive to time mismatches between the RF and envelope input signals. This further proves that the proposed DPD is very general, robust, and effective in a variety of practical applications.

\section{CONCLUSIONS}

A new linearization method for high-efficiency dual-input PA architectures, such as ET and DLM, has been proposed in this paper. A two-step approach is first used to derive the optimal envelope and RF input signals in order to obtain optimal efficiency and linearity performance at the same time. A new dual-input DPD is then developed based on the theoretical derivations and its various applications, e.g., bandwidth reduction for envelope signal and time misalignment are presented. Compared with existing linearization techniques in literature, the proposed method separates the problems of efficiency maximization and distortion minimization, and constructs a more accurate RF input signal for the given envelope signal, thereby making large improvements possible. Experimental results have shown when using the proposed linearization method in the normal operation, the ACLR1 can achieve around $9 \mathrm{~dB}$ improvement compared to the existing linearization methods, while the average PAE is still as high as $49 \%$. When the bandwidth of the envelope signal is reduced, the ACLR1 can still be less than $-45 \mathrm{~dB}$ over a wide bandwidth range. The time mismatch sensitivity is also relaxed if the proposed linearization method is used, which avoids the need for fractional samples delay estimation. Although the proposed linearization method is evaluated on the DLM PA architecture, it can be also applied to other dual-input PA architectures which have similar properties with the DLM 
architecture.

\section{REFERENCES}

[1] F. H. Raab, P. Asbeck, S. Cripps, P. B. Kenington, Z. B. Popović, N. Pothecary, J. F. Sevic, and N. O. Sokal, "Power amplifiers and transmitters for RF and microwave,' IEEE Trans. Microw. Theory Tech., vol. 50, no. 3, pp. 814-826, Mar. 2002.

[2] F. H. Raab, "Intermodulation distortion in Kahn-technique transmitters,' IEEE Trans. Microw. Theory Tech., vol. 44, no. 12, pp. 2273-2278, Dec. 1996.

[3] A. Zhu, P. J. Draxler, C. Hsia, T. Brazil, D. F. Kimball, and P. M. Asbeck, "Digital predistortion for envelope-tracking power amplifiers using decomposed piecewise Volterra series," IEEE Trans. Microw. Theory Tech., vol. 56, no. 10, pp. 2237-2247, Oct. 2008.

[4] F. H. Raab, "High-efficiency linear amplification by dynamic load modulation," in Proc. IEEE MTT-S Int. Microw. Symp. Dig., Jan. 2003, pp. 1717-1720.

[5] H. M. Nemati, C. Fager, U. Gustavsson, and H. Zirath, "An efficiency optimized controlling scheme for dynamic looad modulation of powe amplifiers ," in Proc. 38th Eur. Microw. Conf., Oct. 2008, pp. 583-586.

[6] A. Soltani, H. M. Nemati, H. Cao, T. Eriksson, and C. Fager, "Dynamic load modulation of high power amplifiers with varactor-based matching networks," in Proc. IEEE MTT-S Int. Microw. Symp. Dig., Jun. 2009, pp. 1537-1540.

[7] J. Jeong, D. F. Kimball, M. Kwak, C. Hsia, P. Draxler, and P. M. Asbeck, "Wideband Envelope Tracking Power Amplifiers With Reduced Bandwidth Power Supply Waveforms and Adaptive Digital Predistortion Techniques," IEEE Trans. Microw. Theory Tech., vol. 57, no. 12, pp. 3307-3314, Dec. 2009.

[8] D. Rudolph, "Kahn EER technique with single-carrier digital modulations," IEEE Trans. Microw. Theory Tech., vol. 51, no. 2, pp. 548-552, Feb. 2003

[9] F. Wang, A. H. Yang, D. F. Kimball, L. E. Larson, and P. M. Asbeck, "Design of wide-bandwidth envelope-tracking power amplifiers for OFDM applications," IEEE Trans. Microw. Theory Tech., vol. 53, no. 4, pp. 1244-1255, Apr. 2005.

[10] H. Cao, H. M. Nemati, A. S. Tehrani, T. Eriksson, J. Grahn, and C. Fager, "Linearization of efficiency-optimized dynamic load modulation transmitter architectures," IEEE Trans. Microw. Theory Tech., vol. 58, no. 4, pp. 873-881, Apr. 2010.

[11] H. M. Nemati, H. Cao, B. Almgren, T. Eriksson, and C. Fager, "Design of Highly Efficient Load Modulation Transmitter for Wideband Cellular Applications," IEEE Trans. Microw. Theory Tech., vol. 58, no. 11, pp. 2820-2828, Nov. 2010.

[12] A. Hekkala, A. Kotelba, M. Lasanen, P. Järvensivu, and A. Mämmelä, "Novel Digital Compensation Approaches for Envelope Tracking Amplifiers," To Appear in Wireless Pers. Commun., 2010.

[13] I. Kim, Y. Y. Woo, J. Kim, J. Moon, J. Kim, and B. Kim, "HighEfficiency Hybrid EER Transmitter Using Optimized Power Amplifier,' IEEE Trans. Microw. Theory Tech., vol. 56, no. 11, pp. 2582-2593, Nov. 2008.

[14] I. Kim, J. Kim, J. Moon, and B. Kim, "Optimized Envelope Shaping for Hybrid EER Transmitter of Mobile WiMAX-Optimized ET Operation," IEEE Trans. Microw. Wireless Compon. Lett., vol. 19, no. 5, pp. 335-337, May 2009.

[15] J. H. Qureshi, M. J. Pelk, M. Marchetti, W. C. E. Neo, J. R. Gajadharsing, M. P. van der Heijden, and L. C. N. de Vreede, "A 90-W Peak Power GaN Outphasing Amplifier with Optimum Input Signal Conditioning," IEEE Trans. Microw. Theory Tech., vol. 57, no. 8, pp. 1925-1935, Aug. 2009.

[16] M. Schetzen, The Volterra and Wiener Theories of Nonlinear Systems. Malabar, FL: Krieger, 2006.

[17] H. Cao, A. Soltani, H. M. Nemati, C. Fager, T. Eriksson, and H. Zirath, "Time Alignment in a Dynamic Load Modulation Transmitter Architecture," in Proc. 39th Eur. Microw. Conf., Oct. 2009, pp. 1211-1214.

[18] A. Cesari, A. Cid-Pastor, C. Alonso, and J.-M. Dilhac, "A DSP structure authorizing reduced-bandwidth DC/DC Converters for Dynamic Supply of RF Power Amplifiers in Wideband Applications," in Proc. Industrial Electronics. Conf., Nov. 2006, pp. 3361-3366.

[19] G. Montoro, P. L. Gilabert, E. Bertran, and J. Berenguer, "A method for real-time generation of slew-rate limited envelopes in envelope tracking transmitters," in IEEE Int. Microw. Series on RF Front-ends for Soft. Defined and Cognitive Radio Solutions, Feb. 2010, pp. 1-4.

[20] G. Montoro, P. L. Gilabert, J. Berenguer, and E. Bertran, "Digital predistortion of envelope tracking amplifiers driven by slew-rate limited envelopes," in Proc. IEEE MTT-S Int. Microw. Symp. Dig., Jun. 2011, pp. 1-4.

[21] D. R. Morgan, Z. Ma, J. Kim, M. G. Zierdt, and J. Pastalan, "A Generalized Memory Polynomial Model for Digitial Predistortion of RF Power Amplifiers," IEEE Trans. Signal Process., vol. 54, no. 10, pp. 3852-3860, Oct. 2006.

[22] A. S. Tehrani, H. Cao, S. Afsardoost, T. Eriksson, M. Isaksson, and C. Fager, "A Comparative Analysis of the Complexity/Accuracy Tradeoff in Power Amplifier Behavioral Models," IEEE Trans. Microw. Theory Tech., vol. 58, no. 6, pp. 1510-1520, Jun. 2010.

[23] “3GPP Specification TS 25.141," Base Station Conformance Testing (FDD), 2000.

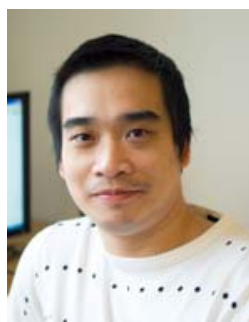

Haiying Cao (S'10) received the B.E. degree in communication engineering from Beijing University of Posts and Telecommunications, Beijing, China, in 2005, the M.Sc. degree in communication engineering and Ph.D. degree from Chalmers University of Technology, Göteborg, Sweden, in 2007 and 2011, respectively.

$\mathrm{He}$ is currently working as a research engineer in Ericsson AB, Kista, Sweden. His research interests include advanced digital signal processing in wireless communication systems, behavioral modeling for RF power amplifiers, nonlinear system identification algorithms and highefficiency power amplifier architectures.

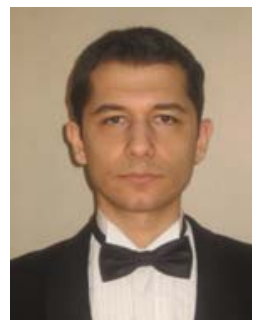

Hossein Mashad Nemati (S'07) was born in Tehran, Iran, in 1980. He received the B.Sc. degree in telecommunication engineering from the Amirkabir University of Technology, Tehran, Iran, in 2004, the M.Sc. and Ph.D. degrees in microwave electronics from the Chalmers University of Technology, Göteborg, Sweden, in 2006 and 2010, respectively.

He is currently working with research and development of power amplifiers at Ericsson AB, Sweden.

His research interests are high-efficiency PAs and transmitter architectures. Dr. Nemati was the recipient of the 2008 Outstanding Achievement Award, and the 2010 First Place Award of the Student High Efficiency Power Amplifier Design Competition of the IEEE Microwave Theory and Techniques Society (IEEE MTT-S) International Microwave Symposium (IMS).

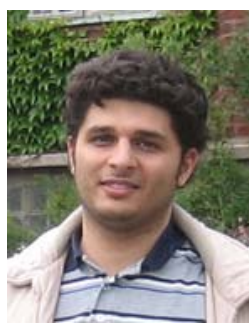

architectures.
Ali Soltani Tehrani (S'09) received his B.Sc degree in communication engineering from K.N. Toos University of Technology, Tehran, Iran in 2005. $\mathrm{He}$ received the M.Sc degree in communication engineering from Chalmers University of Technology, Sweden, in 2007. He is currently working towards his Ph.D. degree in Chalmers University of Technology. His research interests currently include utilizing signal processing techniques for hardware impairments, power amplifier behavioral modeling and linearization, and high efficiency transmitte 


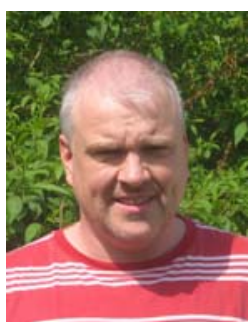

Thomas Eriksson was born on April 7, 1964 in Skövde, Sweden. He received the M.Sc. degree in Electrical Engineering in 1990, and the Ph.D. degree in Information Theory in 1996, both from Chalmers University of Technology, Göteborg, Sweden. He was at AT\&T Labs - Research from 1997 to 1998 , and in 1998 and 1999 he was working on a joint research project with the Royal Institute of Technology and Ericsson Radio Systems AB. From 1999, he is an Associate Professor at Chalmers, and his research interests include communication, vector quantization, speaker recognition, and system modelling of non-ideal hardware components.

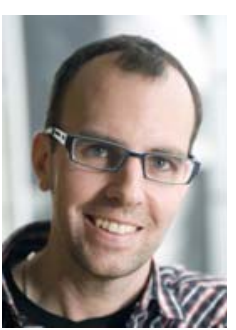

Christian Fager received the M.Sc. and Ph.D. degrees in Electrical Engineering and Microwave Electronics, from Chalmers University of Technology, Sweden, in 1998 and 2003, respectively. He is currently an Associate Professor and project leader in the GigaHertz Centre at the Microwave Electronics Laboratory. In 2002 he received the Best Student Paper Award at the IEEE International Microwave Symposium. His research interests are in the areas of large signal transistor modeling and high efficiency power amplifier architectures. 